\title{
Dural-based Cavernous Malformation at the Temporal Convexity Presenting with Hemorrhage in a Pregnant Woman: Case Report
}

\author{
Kazuki ISHII, ${ }^{1}$ Takafumi TANEI, ${ }^{1}$ Takenori KATO, ${ }^{1}$ Takehiro NAITO, ${ }^{1}$ \\ Eisuke TsuKamoto, ${ }^{1}{ }^{\text {Ko OKADA }},{ }^{1}$ and Toshinori HASEGAWA ${ }^{1}$ \\ ${ }^{1}$ Department of Neurosurgery, Komaki City Hospital, Komaki, Aichi, Japan
}

\begin{abstract}
Intracranial cavernous malformations (CMs) are benign vascular malformations that arise mostly within the brain parenchyma, but occasionally from the dura mater. Here, we report an extremely rare case of a 29-year-old pregnant woman presenting with hemorrhage in a dural-based CM at the temporal convexity. She presented with headache at 38 weeks of pregnancy. Imaging showed a hemorrhagic mass lesion in the left temporal lobe. Consciousness was clear, with no apparent neurological symptoms or headache. Her baby was delivered by emergency Caesarean section. Magnetic resonance (MR) imaging revealed an enhanced lesion around the hematoma and flow void appearances. Cerebral angiography confirmed the left middle meningeal artery feeding the lesion with apparent contrast medium pooling. Surgical removal of the lesion as a single block was performed. Histological examinations were consistent with $\mathrm{CM}$. The lesion was located outside the brain and attached to the dura mater of the convexity, so the final diagnosis was duralbased CM. The patient was discharged home with her baby without any neurological deficits, and no recurrence has been seen for 2 years. Dural-based CM at the temporal convexity was detected, presenting as headache induced by hemorrhage during pregnancy. The relationship between bleeding of the lesion and pregnancy remains unclear, but female hormones and vascular growth factors during pregnancy can induce morphological changes and angiogenesis in CMs.
\end{abstract}

Keywords: dural-based, cavernous malformation, pregnancy, hemorrhage, convexity

\section{Introduction}

Intracranial cavernous malformations (CMs) are benign vascular malformations that account for $5-13 \%$ of central nervous system vascular lesions. ${ }^{11}$ These lesions are mostly found within the brain parenchyma, but have occasionally been reported to arise from the dura mater. The vast majority of dural-based CMs are found in the middle cranial fossa and associated with the cavernous sinus. ${ }^{2)}$ Dural-based CMs outside the middle fossa are rare and arise from the supratentorial convexity, dural sinuses, falx cerebri, tentorium, and superficial posterior fossa. $^{3-9)}$ Symptoms of dural-based CMs

Received April 9, 2020; Accepted May 20, 2020

Copyright $\subseteq 2021$ by The Japan Neurosurgical Society This work is licensed under a Creative Commons AttributionNonCommercial-NoDerivatives International License. outside the middle fossa vary depending on the location and size of the lesion, and can include headaches, seizure, and focal neurological deficits, but rarely include intracranial hemorrhage. ${ }^{5,9)}$ Pregnant women release female hormones and several vascular growth factors that are known to induce morphological changes and angiogenesis of CMs. ${ }^{10,11)}$ These changes are more likely to increase the bleeding from CMs. Here, we report an extremely rare case of a pregnant woman presenting with hemorrhage in a dural-based CM at the temporal convexity.

\section{Case Report}

A 29-year-old woman presented with headache at 38 weeks of pregnancy. Computed tomography (CT) and magnetic resonance (MR) images showed a hemorrhagic mass lesion in the left temporal lobe (Fig. 1A-1C). The patient showed clear consciousness and no apparent neurological symptoms, 

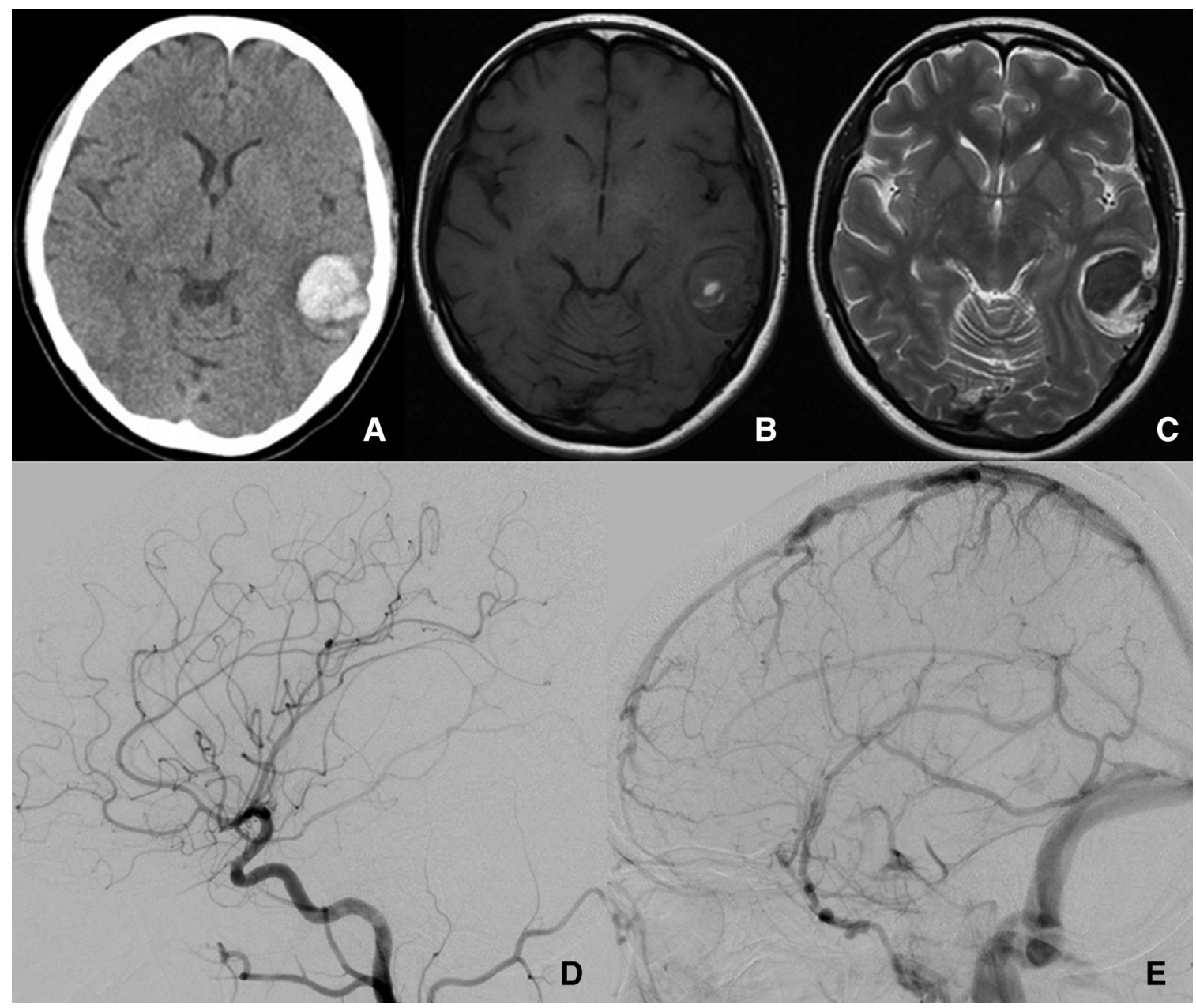

Fig. 1 (A) CT shows hematoma in the left temporal lobe. (B, C) MR images show the hematoma as an iso-intense area on T1-weighted imaging (B), and a low-intensity area on T2-weighted imaging. (D, E) Lateral view of left cerebral angiography after 1 week shows no apparent vascular abnormalities without only slight pooling of contrast medium at the hematoma (D: arterial phase; E: venous phase). CT: computed tomography, MR: magnetic resonance.

including headache. Her baby was delivered by emergency Caesarean section. On cerebral angiography performed 1 week later, vascular abnormalities were not apparent but slight contrast medium was seen pooling at the hematoma (Figs. 1D and $1 \mathrm{E})$. One month later, MR imaging using gadolinium contrast medium and cerebral angiography were performed. MR images revealed an enhanced lesion around the shrinking hematoma and flow void appearances (Fig. 2A). Cerebral angiography confirmed the left middle meningeal artery feeding the lesion with apparent pooling of contrast medium (Figs. $2 \mathrm{~B}$ and $2 \mathrm{C}$ ). These findings indicated that the hemorrhage was induced from some kind of vascular or tumor lesion. We planned surgical removal of the lesion for treatment and confirmation of the pathological diagnosis. Coil embolization of the feeding artery was performed before surgical removal of the lesion (Fig. 2D). Surgical removal of the lesion in a single block was performed via left temporal craniotomy. The lesion had an elastic outer capsule and was attached to the dura mater of the temporal convexity. The lesion was located outside the brain and the hematoma was contained within the lesion, so the brain remained intact (Figs. 2E and $2 \mathrm{~F}$ ). The attachment to the dura mater was also resected, and duraplasty was performed using artificial dura mater. Histological examination revealed a lesion connected to the dura mater and hyperplastic vessels of various sizes (Fig. 3A). The vessels were free of smooth muscle and elastic lamellae. Multiple irregular vascular channels were lined by flattened endothelium in a single cell layer (Fig. 3B). Immunohistochemical analysis showed positive staining for smooth muscle actin (Fig. 3C) and staining for CD34 and CD31 in endothelial cells (Figs. 3D and 3E). Azan staining showed positivity for collagen in stromal tissues (Fig. 3F). These 


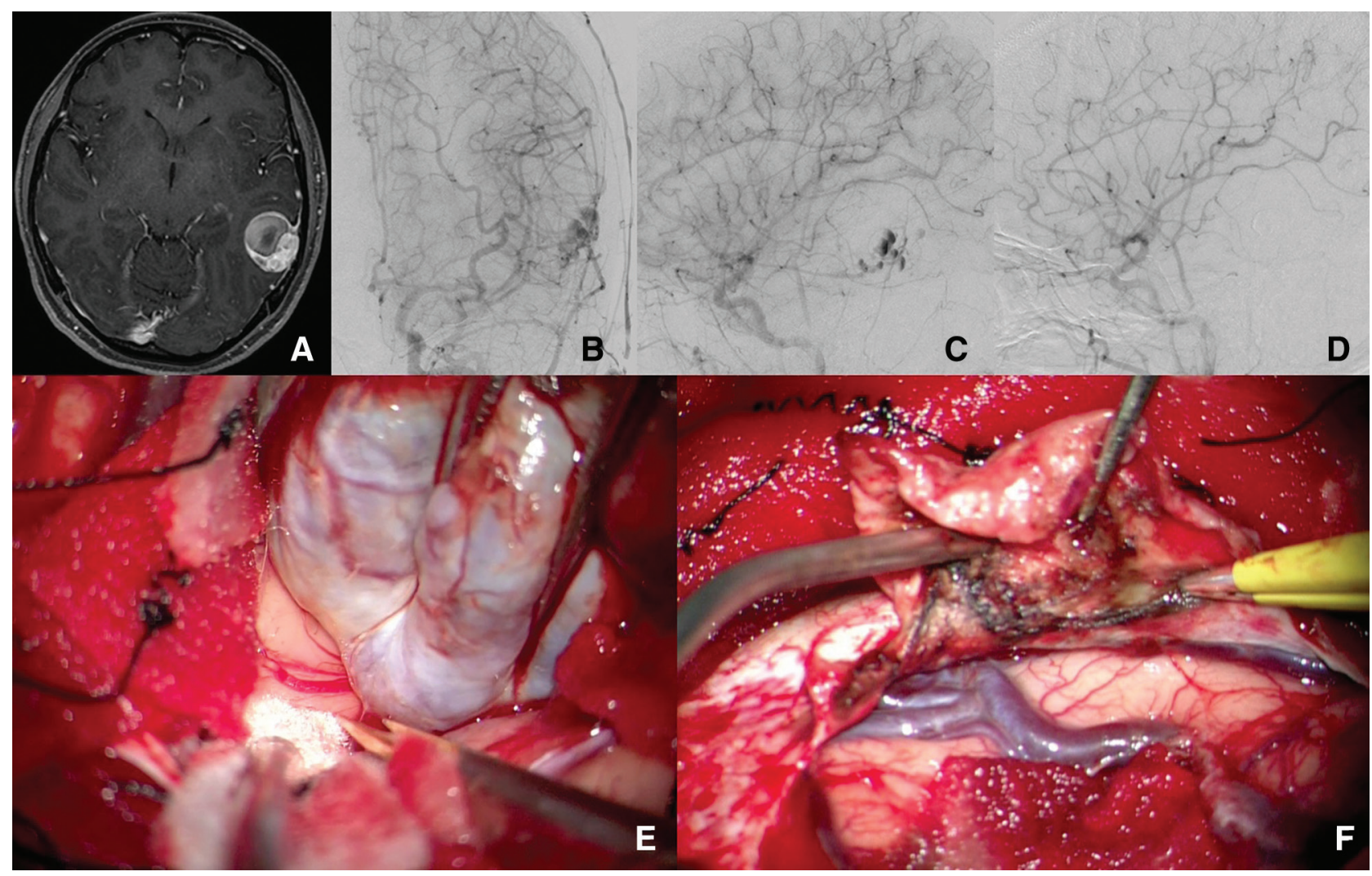

Fig. 2 (A) T1-weighted MR imaging with gadolinium reveals an enhancing lesion around the hematoma and flow void appearances. (B, C) Left carotid angiography after 1 month shows the left middle meningeal artery feeding the lesion with apparent pooling of contrast medium (B: arterial-phase anteroposterior view; C: venousphase lateral view). (D) Lateral view of left carotid angiography after coil embolization shows disappearance of the feeding artery. (E, F) Intraoperative photographs show the lesion with an elastic outer capsule (E), and attached to the dura mater of the temporal convexity, so the brain was intact (F). MR: magnetic resonance.

findings were consistent with CM. The lesion was located outside the brain and attached to the dura mater at the convexity, so the final diagnosis was dural-based CM. The patient was discharged home with her baby without any neurological deficits, and no recurrence has been seen as of the time of writing, 2 years postoperatively.

\section{Discussion}

Intracranial CMs are benign vascular malformations that develop due to slow blood flow across dilated vessels. They consist of sinusoidal vascular spaces lined by a single layer of endothelium. ${ }^{1)}$ Sinusoidal vascular channels of cerebral CMs are located within the brain and are separated by fibrous tissue, but they lack any intervening brain parenchyma or large feeding artery or draining vein. CMs occasionally grow or bleed, depending on endocrine and angiogenic factors. ${ }^{1,9)}$ Intracranial CMs are usually found in brain parenchyma but rarely in extra-cerebral locations. The terminology surrounding CMs has been inconsistently defined. The most recent revision of the International Society for the Study of Vascular Anomalies (ISSVA) classification in 2018 separates vascular anomalies into vascular malformations and vascular tumors. ${ }^{12)}$ Intracranial CMs that used to be called "cavernous (hem)angioma" or "cavernoma" are now classified as "cerebral cavernous malformation" (CCM). CMs arising from the dura mater used to be called "dural-based cavernous (hem)angioma," "dural-based cavernous malformation," "dural cavernous (hem)angioma," or "extra-axial cavernous angioma," ${ }^{-9)}$ These are not currently classified under the category of CCM because they exist outside the brain parenchyma, and are therefore classified as "Other" venous malformations in the ISSVA classification. Histopathologically, CCM and dural-based CM are identical lesions. ${ }^{3)}$ The distinction between the two lesions is based on their differing origins.

Imaging findings for dural-based CM are well known to mimic those of meningioma. Accurate 


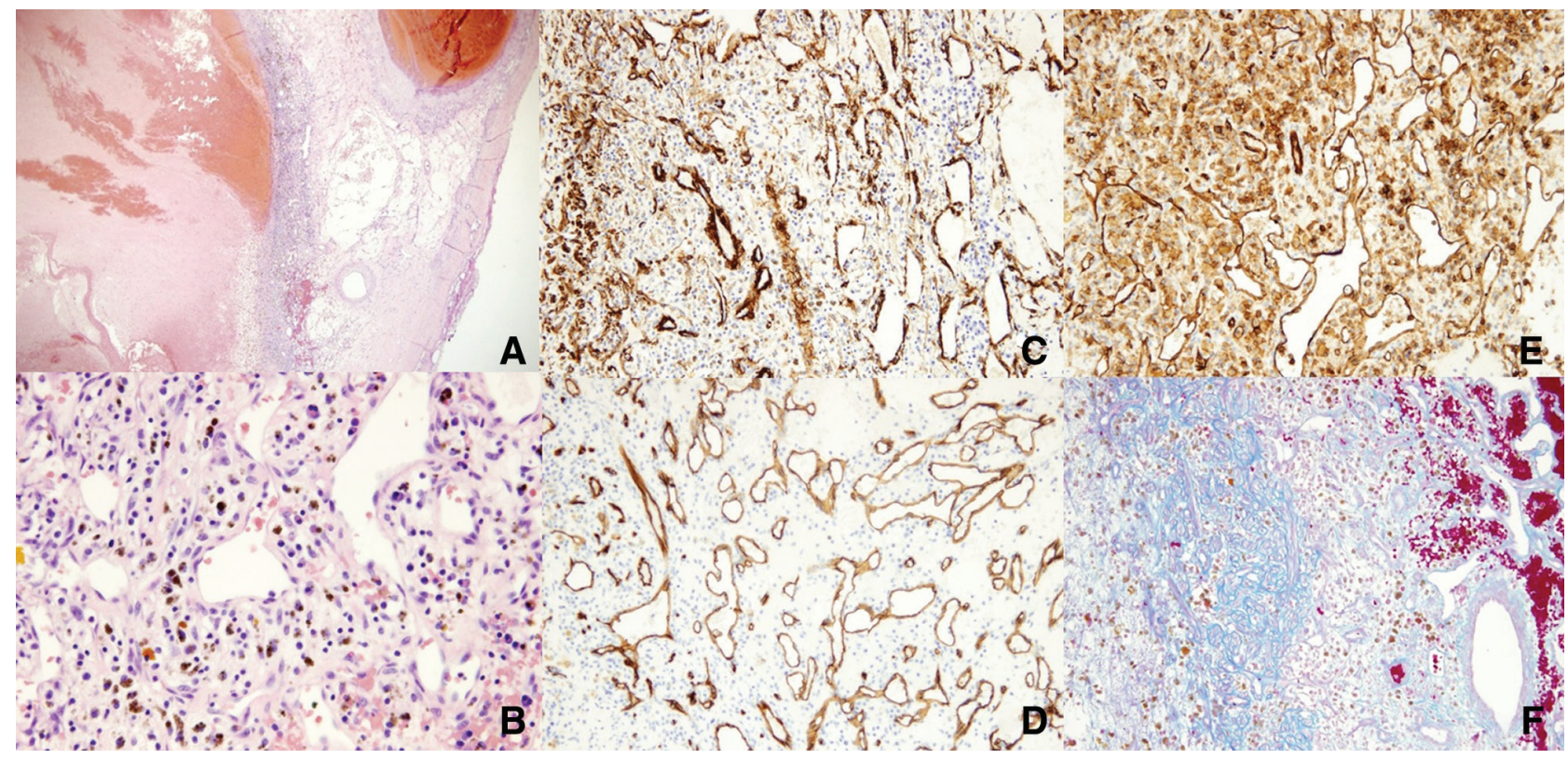

Fig. 3 Histological examinations show the lesion is connected to dura mater and hyperplastic vessels of various sizes. The vessels are free of smooth muscle and elastic lamellae. Multiple irregular vascular channels lined by flattened, single-layer endothelium (hematoxylin-eosin stain; A, $\times 20 ; \mathrm{B}, \times 400$ ). Immunohistochemical examinations show positive staining for smooth muscle actin (C), and staining for CD34 and CD31 in endothelial cells (D: CD34; E: CD31). Azan staining shows positive results for collagen in stromal tissues (F).

preoperative diagnosis of dural-based CM based on preoperative imaging investigations is thus difficult. On MR images, dural-based CMs showed diverse appearances on T1- and T2-weighted imaging. These lesions are well enhanced by gadolinium contrast medium, and sometimes show a dural tail sign similar to that seen in meningiomas. CCMs usually display a peripheral hemosiderin rim to varying degrees, although dural-based CMs do not show such characteristics. ${ }^{13)}$ Cerebral angiography mostly yields negative results, although faint vascular blush can occasionally be seen. ${ }^{13)}$ Presenting symptoms of dural-based CM are usually related to the size and effects on adjacent structures. Hemorrhage is rare, but occasional cases presenting with acute subdural hematoma caused by hemorrhage from a dural-based CM located at the convexity of the dura mater have been reported. ${ }^{5,9)}$ Total surgical removal is the gold standard of treatment for dural-based $\mathrm{CMs}$ and allows definitive diagnosis. When total removal using duraplasty is achieved, adjuvant therapy is unnecessary.

It is well known that not only CMs but also other vascular malformations can exhibit morphological changes under the influence of the major fluctuations in female hormones (including estrogen and progesterone) during pregnancy. ${ }^{10)}$ In addition, increasing blood levels of vascular growth factors, such as vascular endothelial growth factor and basic fibroblast growth factor, can induce angiogenesis and grow in size and increase the likelihood of bleeding from CMs. The relationships between bleeding and growth of dural-based CMs during pregnancy are unclear because these lesions are rare. However, non-hemorrhagic cases during pregnancy have been reported, such as enlargement of duralbased CMs in the middle cranial fossa and a mass lesion in the anterior fossa. ${ }^{11,14)}$ This is the first report of a pregnant woman presenting with hemorrhage in a dural-based CM at the temporal convexity. Of all patients with CMs, only $9 \%$ are diagnosed before 18 years old. Pre-pregnancy diagnosis of intracranial $\mathrm{CM}$ can thus be difficult. ${ }^{15)}$ The use of gadolinium as a contrast agent for MR or cerebral angiography is usually contraindicated by pregnancy because of the potential for adverse effects on the fetus. In addition, surgery for intracranial CMs under general anesthesia in pregnant women can be complicated due to weight gain, increased circulating blood volume, and pregnancy-induced hypertension. It is therefore important to consider the timing and order of imaging examinations, delivery of the child, and surgical removal of the lesion, according to the conditions of the patient and fetus.

Dural-based CMs outside the middle fossa are rare. In this case, dural-based CM at the temporal convexity was detected in a patient presenting with headache induced by hemorrhage during pregnancy. 
The relationship between bleeding of dural-based $\mathrm{CM}$ and pregnancy is unclear; however, female hormones and vascular growth factors during pregnancy can induce morphological changes and angiogenesis for CMs.

\section{Conflicts of Interest Disclosure}

All authors certify that they have no affiliations with or involvement in any organization or entity with any financial interest (e.g., honoraria; educational grants; participation in speakers' bureaus; membership, employment, consultancies, stock ownership, or other equity interest; and expert testimony or patent-licensing arrangements) or non-financial interest (e.g., personal or professional relationships, affiliations, knowledge, or beliefs) in the subject matter or materials discussed in this manuscript.

\section{References}

1) Simard JM, Garcia-Bengochea F, Ballinger WE, Mickle JP, Quisling RG: Cavernous angioma: a review of 126 collected and 12 new clinical cases. Neurosurgery 18: 162-172, 1986

2) Linskey ME, Sekhar LN: Cavernous sinus hemangiomas: a series, a review, and an hypothesis. Neurosurgery 30: 101-108, 1992

3) Biondi A, Clemenceau S, Dormont D, et al.: Intracranial extra-axial cavernous (HEM) angiomas: tumors or vascular malformations? J Neuroradiol 29: 91-104, 2002

4) Bteich F, Kassab C, El Hage G, Moussa R, Abadjian GA, Bou-Nassif R: Atypical presentation of parietal convexity dural-based cavernous hemangioma: a case report and review of literature. World Neurosurg 128: 403-407, 2019

5) Li G, Zhai X, Zhang Y, Liang P, Wu X, Hou K: Dural-based cavernous malformation at the cerebral convexity: report of two pediatric patients. World Neurosurg 112: 81-85, 2018
6) Li Z, Wang C, Ma L, Wu C, Zhao Y, Jiang Z: Multiple nodular dural cavernous angiomas occluding superior sagittal sinus and destructing calvarium: case report and literature review. J Clin Neurosci 58: 218-221, 2018

7) Mansour TR, Medhkour Y, Entezami P, Mrak R, Schroeder J, Medhkour A: The art of mimicry: anterior clinoid dural-based cavernous hemangioma mistaken for a meningioma. World Neurosurg 100: 708.e19-708.e22, 2017

8) Melone AG, Delfinis CP, Passacantilli E, Lenzi J, Santoro A: Intracranial extra-axial cavernous angioma of the cerebellar falx. World Neurosurg 74: 501-504, 2010

9) Suzuki K, Kamezaki T, Tsuboi K, Kobayashi E: Dural cavernous angioma causing acute subdural hemorrhage-case report. Neurol Med Chir (Tokyo) 36: 580-582, 1996

10) Yamada S, Nakase H, Nakagawa I, Nishimura F, Motoyama Y, Park YS: Cavernous malformations in pregnancy. Neurol Med Chir (Tokyo) 53: 555-560, 2013

11) Haber JS, Kesavabhotla K, Ottenhausen M, et al.: Conservative management of cavernous sinus cavernous hemangioma in pregnancy. J Neurosurg 120: 13091312, 2014

12) ISSVA classification for vascular anomalie. https://www. issva.org/UserFiles/file/ISSVA-Classification-2018.pdf. (Accessed on 2020 Feb 1)

13) Vogler R, Castillo M: Dural cavernous angioma: MR features. AJNR Am J Neuroradiol 16: 773-775, 1995

14) Isla A, Roda JM, Alvarez F, Muñoz J, García E, Blázquez MG: Intracranial cavernous angioma in the dura. Neurosurgery 25: 657-659, 1989

15) Kivelev J, Niemelä M, Hernesniemi J: Characteristics of cavernomas of the brain and spine. J Clin Neurosci 19: 643-648, 2012

Corresponding author: Kazuki Ishii, MD Department of Neurosurgery, Komaki City Hospital, 1-20 Jobushin, Komaki, Aichi 485-8520, Japan. e-mail: ishii.kazuki31@gmail.com 